\title{
Comparer des relevés de dates différentes au même emplacement. Exemple du Tchad
}

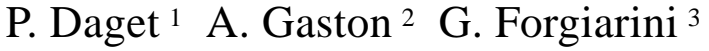

\author{
Mots-clés \\ Pastoralisme - Végétation - \\ Méthode - Tchad.
}

\section{Résumé}

Diverses méthodes sont exposées pour comparer la flore, la végétation et la stratification du tapis végétal d'une station particulière à deux dates successives : application à des steppes du Tchad.

\section{INTRODUCTION}

La végétation sahélienne du Tchad a été étudiée depuis les années 1960 et a fait l'objet d'un suivi plus ou moins régulier, eu égard aux événements survenus dans ce pays. Les observations ont été recueillies selon des protocoles homogènes et ont été enregistrées dans la base Flotrop $(7,13)$. Il nous paraît intéressant d'étudier les variations de composition floristique survenues au cours du temps afin de cerner d'éventuelles évolutions du couvert végétal. La présente note propose un groupe de méthodes simples pour caractériser les éventuelles variations et mesurer les écarts observés entre deux dates. Ces méthodes sont appliquées à la confrontation de couples de relevés extraits de la base.

\section{METHODE D'APPROCHE}

Pour illustrer les méthodes présentées, deux relevés ont été retenus ; ils proviennent des savanes au nord-est du lac Tchad à huit ans d'intervalle. Leurs compositions sont présentées au tableau I. Les listes sont différentes, tant sur les présences que sur les abondancesdominances ; le problème est d'évaluer l'importance de ces différences.

\section{Tableau I}

Deux relevés du Tchad faits au même emplacement avec dix ans d'écart

\begin{tabular}{lcc} 
Espèces & Relevé $\mathbf{1}$ & Relevé 2 \\
\hline Abildgaardia hispidula & $0^{*}$ & 1 \\
Acacia raddiana & 0 & 0 \\
Acacia senegal & 0 & 0 \\
Aristida mutabilis & 4 & 3 \\
Balanites aegyptiaca & 0 & 0 \\
Cenchrus biflorus & 0 &. \\
Dactyloctenium aegyptium &. & 0 \\
Eragrostis tremula &. & 1 \\
Gisekia pharnacoides &. & 0 \\
Ipomoea coptica & 1 & 0 \\
Leptadenia pyrotechnica & 0 & 3
\end{tabular}

* Les coefficients d'abondance-dominance selon l'échelle classique (Boudet, 1970, Iemvt ; Braun-Blanquet, 1963, Springer) dans laquelle le symbole + est remplacé par 0

\section{Différence entre flores}

En ce qui concerne la flore, le problème est aisé, il suffit de calculer la distance de Hamming entre ces deux relevés ; on sait (4) qu'elle s'écrit:

$\mathrm{H}=1-\mathrm{J}$

où $\mathrm{J}$ est le coefficient de communauté de Jaccard égal au rapport du nombre d'espèces communes au nombre total d'espèces. Ici, sur 11 espèces, sept sont communes entre les deux relevés, donc :

$\mathrm{H}=100\left(1-\frac{7}{11}\right)=36$
1. Cirad-emvt et Grefo, TA 30/E, campus international de Baillarguet, 34398 Montpellier Cedex 5, France

2. UMR d'agronomie, Inra-Ina.PG, BP 01, 78850 ThivernaL-Grignon, France Actuellement : résidence Métropole D7-99, av. du Général Leclerc, 94700 Maisons-Alfort, France

3. Cirad-emvt, TA 30/E, 34398 Montpellier Cedex 5, France forgiarini@cirad.fr 
On retiendra les qualifications suivantes :

- différence floristique très faible pour $\mathrm{H}<20$

- faible $20 \leq \mathrm{H}<40$

- moyenne $40 \leq \mathrm{H}<60$

- forte $60 \leq \mathrm{H}<80$

- très forte $80 \leq \mathrm{H}$

Ici, avec $\mathrm{H}=36$ compris entre 20 et 40 , la différence floristique est faible.

\section{Différence entre végétations}

Pour comparer la masse végétale, on utilisera la distance $\mathrm{D}$ donnée par le complément à 1 du coefficient de communauté de Kulczinski $(1,8)$ :

$\mathrm{D}=1-\mathrm{K}$

$\mathrm{K}$ s'exprimant comme la somme des contributions spécifiques $(\mathrm{Cs})$ minimales des espèces communes. Dans une première étape, il faut donc convertir les coefficients d'abondance-dominance en recouvrements. Cette conversion est faite (6) par la formule :

$\mathrm{R}=\operatorname{IND}\left(\frac{90}{3^{5-c}}\right)$

où $\mathrm{c}$ est le coefficient d'abondance-dominance, avec la fonction $\operatorname{IND}(\mathrm{x})$ qui extrait la partie entière de tout $\mathrm{x}$; de plus, on pose que $\mathrm{R}=0,1$ quand $\mathrm{c}=0$. Le tableau I (colonnes 2 et 3 ) devient tableau II avec les contributions spécifiques (8) figurant dans les colonnes 4 et 5 ; rappelons que les contributions spécifiques s'obtiennent à partir des recouvrements par :

$\mathrm{Cs}(\mathrm{i})=100 \frac{\mathrm{R}(\mathrm{i})}{\sum_{\mathrm{I}=1}^{\mathrm{I}=\mathrm{n}} \mathrm{R}(\mathrm{i})}$

donc, pour Abildgaardia hispidula dans le relevé 2, il vient :

$\operatorname{Cs}(1)=100 \frac{1}{1+0,1+\ldots+10}=4,4$

\section{Tableau II}

Recouvrements et contributions spécifiques calculés d'après les coefficients d'abondancedominance dans les deux relevés du Tchad

\begin{tabular}{lccccc} 
& \multicolumn{2}{c}{ Recouvrement } & \multicolumn{2}{c}{ Contribution } \\
\cline { 2 - 3 } \cline { 5 - 6 } & $\mathbf{1}$ & $\mathbf{2}$ & $\mathbf{1}$ & $\mathbf{2}$ \\
\hline Abildgaardia hispidula & 0,1 & 1 & $\mathbf{0 , 3}$ & 4,4 \\
Acacia raddiana & 0,1 & 0,1 & $\mathbf{0 , 3}$ & 0,4 \\
Acacia senegal & 0,1 & 0,1 & $\mathbf{0 , 3}$ & 0,4 \\
Aristida mutabilis & 30,0 & 10,0 & 94,9 & $\mathbf{4 4 , 2}$ \\
Balanites aegyptiaca & 0,1 & 0,1 & $\mathbf{0 , 3}$ & 0,4 \\
Cenchrus biflorus & 0,1 &. & 0,3 & 0 \\
Dactyloctenium aegyptium & $\cdot$ & 0,1 & $\mathbf{0}$ & 0,4 \\
Eragrostis tremula & $\cdot$ & 1 & $\mathbf{0}$ & 4,4 \\
Gisekia pharnacoides & $\cdot$ & 0,1 & $\mathbf{0}$ & 0,4 \\
Ipomoea coptica & 1 & 0,1 & 3,2 & $\mathbf{0 , 4}$ \\
Leptadenia pyrotechnica & 0,1 & 10 & $\mathbf{0 , 3}$ & 44,2
\end{tabular}

En gras : contributions spécifiques minimales

La somme des Cs minimales, indiquées en gras dans le tableau II, est :

$0,3+0,3+0,3+44,2+0,3+0+0+0+0+0,4+0,3=46,1$ donc 46 p. 100 de la masse végétale est restée identique d'une observation à l'autre ; la distance D est dans ce cas : 100 - 46,1 $=53,9 \approx 54$. On utilisera les seuils précédents pour qualifier les divergences observées :

- divergence très faible pour $\mathrm{D}<20$

- faible $20 \leq \mathrm{D}<40$

- moyenne $40 \leq \mathrm{D}<60$

- forte $60 \leq \mathrm{D}<80$

- très forte $80 \leq \mathrm{D}$

Ici, où $\mathrm{D}=54$, la divergence entre les végétations est moyenne.

Il faut noter que la confrontation porte sur la seule masse végétale et non sur la manière dont elle est constituée et sa disponibilité. Aussi, une troisième analyse s'impose-t-elle ; elle porte sur la stratification de la végétation.

\section{Différence entre stratifications}

Les espèces sont réparties en quatre « strates »: arbres, arbustes, herbacées pérennes et annuelles, puis les recouvrements sont cumulés dans chaque strate (tableau III).

\section{Tableau III}

Recouvrements des quatre "strates » dans les deux relevés du tableau I

$\begin{array}{lcc}\text { Arbres } & 0,3 & 0,3 \\ \text { Arbustes } & 0,1 & 10 \\ \text { Pérennes } & 0 & 0 \\ \text { Annuelles } & 31,1 & 12,3\end{array}$

Soient les deux observations $\mathrm{O} 1$ et $\mathrm{O} 2$ d'une des strates faites à deux dates successives au même endroit : on calcule $(5,15)$ leur différence $d$ et leur somme s, puis la statistique :

$\mathrm{X}=\frac{(\mathrm{O} 1-\mathrm{s} / 2)^{2}}{\mathrm{~s} / 2}+\frac{(\mathrm{O} 2-\mathrm{s} / 2)^{2}}{\mathrm{~s} / 2}=\frac{\mathrm{d} 2}{\mathrm{~s}}$

$\mathrm{X}$ suit une loi du $\chi^{2}$ à un degré de liberté. Il y a donc un écart peu significatif (seuil 10 p. 100) entre $\mathrm{O} 1$ et $\mathrm{O} 2$ lorsque $2,71 \leq \mathrm{X}$ $<3,84$, significatif (seuil 5 p. 100 lorsque $3,84 \leq X<6,60$, et très significatif (seuil 1 p. 100) Iorsque $6,60 \leq X$. Dans le cas présent les différentes valeurs son réunies dans le tableau IV.

\section{Tableau IV}

Analyse des différences entre stratifications des relevés du tableau I

$\begin{array}{lcclc} & \text { d } & \text { s } & \text { X } & \text { Signification } \\ \text { Arbres } & 0 & 0,6 & 0 & \mathrm{~ns} \\ \text { Arbustes } & 9,9 & 10,1 & 9,7 & * * * \\ \text { Pérennes } & 0 & 0 & 0 & \mathrm{~ns} \\ \text { Annuelles } & 18,8 & 43,4 & 8,14 & * * *\end{array}$

En huit ans il y eu un embroussaillement très significatif de la station et une diminution drastique du tapis herbacé (rien à signaler quant aux arbres).

Pour l'ensemble des quatre strates, on compare $\mathrm{X}^{\prime}=\mathrm{X} 1+\mathrm{X} 2$ $+\mathrm{X} 3+\mathrm{X} 4$ aux valeurs liminaires $6,25-7,81$ et 11,34 du $\chi^{2}$ à trois degrés de liberté pour décider du niveau de signification des différences de stratification observées (15).

Ici $X^{\prime}=0+9,7+0+8,14=17,84$ 
Comme X' est supérieur au seuil le plus élevé $(11,34)$, la conclusion est qu'en huit ans la divergence entre les stratifications d'une observation à l'autre a été très significative.

\section{Application}

Treize stations ont été repérées dans une zone située entre 13 et $16^{\circ}$ de latitude $\mathrm{N}$ et entre 14 et $18^{\circ}$ de longitude $\mathrm{E}$; il s'agit donc d'une zone s'étendant au nord-est du lac Tchad. Ces stations ont fait l'objet de deux observations à quelques années de distance. Ces observations ont été comparées selon les méthodes précédentes.

- Observations d'A. Gaston, respectivement en 1967 et 1975

Station 1, relevés 249 et 749

Flore : divergence faible $(\mathrm{H}=33)$

Végétation : divergence forte $(\mathrm{D}=77)$

Stratification : différence peu significative au niveau global $\left(X^{\prime}=7\right)$ malgré une augmentation significative des ligneux bas $(X=4,95)$

Station 2, relevés 269 et 753

Flore : divergence forte $(\mathrm{H}=70)$

Végétation : divergence moyenne ( $\mathrm{D}=43)$

Stratification : différence significative au niveau global $\left(X^{\prime}=9,65\right)$ due à un accroissement significatif des annuelles $(X=5,7)$

Station 3, relevés 270 et 754

Flore : divergence très forte $(\mathrm{H}=81)$

Végétation : divergence forte $(\mathrm{D}=79)$

Stratification : divergence globale très significative $\left(X^{\prime}=12,3\right)$, consécutive à une augmentation significative des ligneux bas $(X=5)$, concomitante avec une diminution significative des herbacés pérennes $(X 5,05)$

Station 4, relevés 253 et 764

Flore : divergence très forte $(\mathrm{H}=84)$

Végétation : divergence très forte $(\mathrm{D}=88)$

Stratification : divergence globale significative $\left(X^{\prime}=8,7\right)$, consécutive à une augmentation très significative des ligneux bas $(\mathrm{X}=8,1)$

Station 5, relevés 153 et 772

Flore : divergence complète $(\mathrm{H}=1)$

Végétation : divergence complète $(\mathrm{D}=100)$

Stratification : les divergences observées ne sont pas significatives $\left(X^{\prime}=4,1\right.$ et $\left.X=2,4\right)$

Station 6, relevés 153 et 773

Flore : divergence forte $(\mathrm{H}=79)$

Végétation : divergence très forte $(\mathrm{D}=96)$

Stratification : les divergences observées ne sont pas significatives $\left(X^{\prime}=3,45\right.$ et $\left.X=2,4\right)$

Station 7, relevés 155 et 775

Flore : divergence forte $(\mathrm{H}=62)$

Végétation : divergence moyenne $(\mathrm{D}=51)$

Stratification : divergence globale peu significative $\left(X^{\prime}=6,3\right)$ liée à une divergence des annuelles elle-même peu significative $(X=3,80)$

Station 8 , relevés 309 et 758

Flore : divergence forte $(\mathrm{H}=62)$

Végétation : divergence moyenne $(\mathrm{D}=49)$

Stratification : divergence globale peu significative $\left(X^{\prime}=6,3\right)$ liée à une divergence des annuelles elle-même peu significative $(X=3,80)$

Station 9, relevés 169 et 760

Flore : divergence moyenne $(\mathrm{H}=55)$

Végétation : divergence moyenne $(\mathrm{D}=46)$

Stratification : divergence globale peu significative $\left(X^{\prime}=6\right)$
Station 10 , relevés 314 et 751

Flore : divergence forte $(\mathrm{H}=78)$

Végétation : divergence faible $(\mathrm{D}=28)$

Stratification : divergence globale très significative $\left(X^{\prime}=15,9\right)$ résultant d'un embroussaillement significatif $(X=4,95)$ et d'une chute des annuelles très significative $(X=10,9)$

- Observations respectivement dans les relevés d'A. Gaston en 1967 et d'I. Yosko en 1987

Station 11, relevés 241 et 71

Flore : divergence très forte $(\mathrm{H}=80)$

Végétation : divergence faible $(\mathrm{D}=26)$

Stratification : divergence globale significative $\left(X^{\prime}=9,5\right)$ liée à une diminution significative des herbacées pérennes $(X=6,5)$

Station 12, relevés 240 et 73

Flore : divergence totale $(\mathrm{H}=100)$

Végétation : divergence très faible $(\mathrm{D}<20)$

Stratification : divergence très significative $\left(X^{\prime}=56\right)$ correspondant à un fort embroussaillement $(X=14,9)$ et une augmentation considérable des annuelles $(X=40,7)$

Station 13, relevés 244 et 70

Flore : divergence très forte $(\mathrm{H}=68)$

Végétation : divergence très faible $(\mathrm{D}<20)$

Stratification : divergence très significative $\left(X^{\prime}=13,5\right)$ correspondant à un fort embroussaillement

\section{RESULTATS ET DISCUSSION}

Le tableau V récapitule les résultats obtenus. Il permet de caractériser l'évolution d'ensemble de la végétation, dans la mesure où les couples étudiés en sont représentatifs. Un procédé empirique (9), consistant à caractériser le potentiel évolutif des différentes strates par le nombre d'astérisques qui leur correspondent, peut être employé pour caractériser la dynamique de la végétation :

- pour les arbres, aucun astérisque, pas d'évolution perceptible au cours de la période étudiée ;

- pour les arbustes, 13 astérisques pour un maximum possible de $39(13 \times 3)$, soit 31 p. 100 ; cela traduit une nette tendance à l'embroussaillement des steppes régionales ;

\section{Tableau V}

Analyse de la variation de la stratification dans 13 sites du nord-est du lac Tchad

\begin{tabular}{|c|c|c|c|c|c|}
\hline Paires & Arbres & Arbustes & Pérennes & Annuelles & Ensemble \\
\hline 1 & ns & $* *$ & ns & ns & $*$ \\
\hline 2 & ns & ns & ns & $* *$ & $* *$ \\
\hline 3 & ns & $* *$ & $* *$ & ns & $* * *$ \\
\hline 4 & ns & $* * *$ & ns & ns & $* *$ \\
\hline 5 & ns & $\mathrm{ns}$ & ns & ns & ns \\
\hline 6 & ns & ns & ns & ns & ns \\
\hline 7 & ns & ns & ns & $*$ & $*$ \\
\hline 8 & $\mathrm{~ns}$ & ns & ns & $*$ & $*$ \\
\hline 9 & ns & ns & ns & ns & ns \\
\hline 10 & $\mathrm{~ns}$ & $* *$ & ns & $*$ & $*$ \\
\hline 11 & ns & ns & $* *$ & ns & $* *$ \\
\hline 12 & ns & $* * *$ & ns & $* * *$ & $* * *$ \\
\hline 13 & ns & $* * *$ & ns & $\mathrm{ns}$ & $* * *$ \\
\hline
\end{tabular}


- pour les herbacées pérennes, 4 astérisques, soit 11 p. 100, traduisant une faible diminution des espèces herbacées pérennes qui de toutes manières n'avaient qu'un faible recouvrement global initial ; - pour les annuelles, 16 astérisques, soit 33 p. 100, traduisant une importante variabilité ; le plus souvent il s'agit d'une sensible décroissance de leur recouvrement global, sauf dans un cas où on l'observe une croissance considérable (probablement lié à un aléa climatique).

En définitive, les steppes régionales montrent dans la période 1967-1975 une tendance nette à la dégradation par embroussaillement (10), essentiellement par Leptadenia pyrotechnica qui, à la suite de la sécheresse de 1973 (11, 12, 14), semble avoir occupé une niche écologique rendue disponible. Cet embroussaillement est encore plus net entre 1967 et 1985. Dans certains des peuplements presque purs de cette espèce, on a observé après 1980 que pratiquement tous les individus étaient morts sur pied.

On a parfois cherché à caractériser la dégradation des formations sahéliennes par la proportion de sol nu (SN), cet accroissement traduisant la désertification du Sahel consécutif à la péjoration du climat. L'importance du sol nu peut être évaluée par le complément à 100 de recouvrement global de la végétation, ce dernier étant donné comme la somme des recouvrements de toutes les espèces, ce qui dans ces végétations ouvertes conserve un sens.

$\mathrm{SN}=1-\sum \mathrm{R}(\mathrm{i})$

où $\mathrm{R}(\mathrm{i})$ est le recouvrement de l'espèce i. Cette mesure ne constitue qu'une approximation puisque les plantes herbacées peuvent végéter sous les végétaux ligneux, au moins partiellement, ce qui augmenterait d'autant la proportion de sol nu. L'accroissement réel du sol nu est donc au moins égal à la mesure SN qui est proposée ici. Les valeurs stationnelles de SN pour les diverses stations étudiées ici avant 1970 ont été confrontées avec les mesures correspondantes après 1975 (figure 1). Il n'y a pas de liaison entre l'importance du sol nu mesurée par SN avant ou après la sécheresse de 1974.

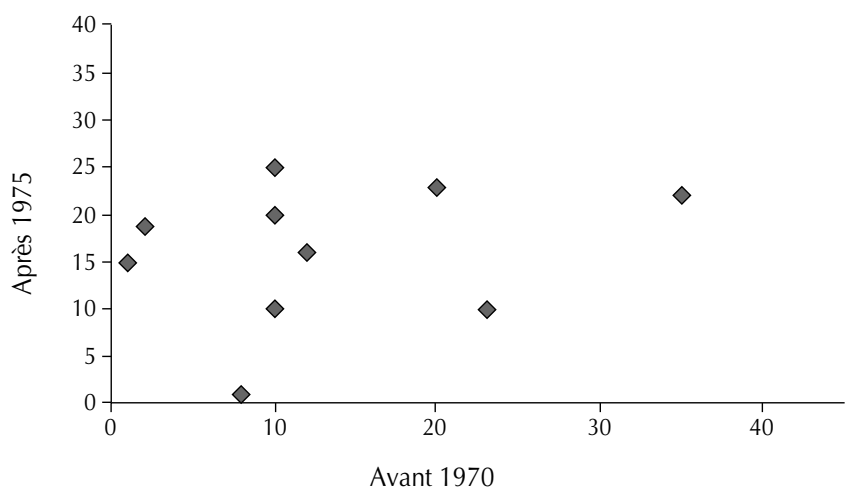

Figure 1 : comparaison des recouvrements globaux avant 1970 et après 1975 dans des steppes tchadiennes au nordest du lac Tchad.

Il est possible de reprocher à la mesure $\mathrm{SN}$ d'inclure les strates ligneuses et de lui préférer une mesure qui ne prendrait en compte que les strates herbacées, les deux dernières du tableau $\mathrm{V}$, parce que ce sont celles qui couvrent directement le sol et donc qui lui assurent protection contre l'érosion surtout éolienne.

\section{BIBLIOGRAPHIE}

1. BECKING R., 1957. The Zurich-Montpellier school of phytosociology. Bot. Rev., 23: 411-488.

2. BOUDET G., 1991. Manuel sur les pâturages tropicaux et les cultures fourragères. Paris, France, La Documentation française, 266 p.

3. BRAUN-BLANQUET J., 1963. Pjlanzensoziologie. Berlin, RFA Springer, $650 \mathrm{p}$

4. CESAR J., DAGET P., 1997. Rechercher des limites écologiques dans une végétation. Revue Elev. Méd. vét. Pays trop., 50 : 153-156.

5. COX D., LEWIS P., 1969. L'analyse statistique des séries d'événements. Paris, France, Dunod, $272 \mathrm{p}$.

6. DAGET P., 1998. Biodiversité. V. La végétation stationnelle. Flotrop Info, $24: 1-2$

7. DAGET P., GASTON A., 1999. La mémoire des pâturages africains. Sécheresse, 10 : 171-182.

8. DAGET P., POISSONET J., 1969. Analyse phytologique des prairies. Applications agronomiques. Montpellier, France, Cnrs-CEPE, 76 p. (Doc. $n^{\circ} 48$ )

9. DAGET-BERTOLETTI L., DAGET P., 1974. Expression empirique de la sensibilité des espèces dans une prairie permanente soumise à des traitements comportant divers niveaux. Fourrages, 57 : 121-131.

\section{Summary}

Daget P., Gaston A., Forgiarini G. Comparing Relevés of Different Dates on the Same Site. Example from Chad

Various methods are described to compare the flora, the vegetation and the stratification of the vegetation cover of a particular station at two successive dates: application to steppes of Chad.

10. DONANGMBAYE M., 1998. Une dégradation peu connue : I'embuissonnement des parcours pastoraux des savanes soudaniennes. Revue sci. Tchad.

11. GASTON A., 1981. La végétation du Tchad (nord-est et sud-est du lac Tchad. Evolution récente sous des influences climatiques et humaines. Thèse Etat, université Pierre et Marie Curie, Paris, France, 333 p.

12. GASTON A., 1991. Rapport de mission auprès du réseau d'observation des pâturages naturels, Ropanat. Maisons-Alfort, France, Ciradlemvt, $34 \mathrm{p}$.

13. GASTON A., DAGET P., 2000. La base Flotrop et l'inventaire de la flore des pâturages d'Afrique sahélienne. In XVIe Congrès international de l'AETFAT, Meise, Belgique.

14. GASTON A., DULIEU D., 1976. Effets de la sécheresse de 1973 sur les pâturages du Kanem Lac. Comparaison avec les études de 19641965. Actualisation de la carte. Maisons-Alfort, France, lemvt, 173 p.

15. SNEDECOR G., COCHRAN W., 1968. Statistical methods. Ames, lowa, IA, USA, lowa University Press, 548 p.

Accepté le 26.02.2004

\section{Resumen}

Daget P., Gaston A., Forgiarini G. Comparar tomas de fechas diferentes en un mismo sitio. Ejemplo del Chad

Se exponen diversos métodos para comparar la flora, la vegetación y la estratificación de la cobertura vegetal de una estación en particular en dos fechas sucesivas: aplicación a estepas de Chad.

Palabras clave: Pastoralismo - Vegetación - Método - Chad. 